\title{
Testing Exponentiality by comparing the Empirical Distribution Function of the Normalized Spacings with that of the Original Data
}

\author{
S.Rao Jammalamadaka \\ Department of Statistics and Applied Probability \\ University of California \\ Santa Barbara, CA 93106-3110 \\ Emanuele Taufer \\ Department of Computer and Management Sciences \\ University of Trento \\ Trento, I 38100.
}

Summary. We introduce new goodness of fit tests for exponentiality by using a characterization based on normalized spacings. We provide relevant asymptotic theory for these tests and study their efficiency. An empirical power study and comparisons are also provided.

Keywords Test for exponentiality, Kolmogorov-Smirnov statistics, Omega square statistics, Brownian Bridge, Asymptotic efficiency. 


\section{Introduction}

In this paper we develop a goodness of fit test for exponentiality exploiting a characterization based on the "normalized spacings." There is considerable literature on the problem of testing for exponentiality. The reasons are many-fold and chief among these are: the watershed role played by the exponential distribution in reliability and survival analysis, its nice mathematical properties, as well as the availability of several characterizations. Most recently, Baringhaus and Henze (2000) and Taufer (2000) developed tests based on the mean residual life characterization. Alwasel (2001), Ahmad and Alwasel (1999) exploit a characterization based on the lack of memory of the exponential distribution. This property is also the basis for tests considered earlier by Angus (1982) who uses Kolmogorov-Smirnov and Cramer-von Mises type test statistics based on the difference of the empirical distribution functions $\bar{F}_{n}(2 x)$ and $\bar{F}_{n}^{2}(x)$, where $\bar{F}=1-F$, denotes the survival function. Grzegorzewsi and Wieczorkowski (1999) and Ebrahimi et Al. (1992) make use of the maximum entropy characterization by considering the difference between a nonparametric estimator of entropy and the maximum likelihood estimator of entropy under exponentiality. Other omnibus tests for exponentiality have been developed by Henze (1993), Baringhaus and Henze (1991, 1992) who used weighted distance measures between sample estimators of the Laplace transform and its counterpart under the null hypothesis. Klar (2000) provides a test against Harmonic New Better than Used in Expectation (HNBUE) alternatives by using estimates of $\int_{x}^{\infty} \bar{F}(t) d t-\theta e^{-x / \theta}$ based on the empirical distribution function and the sample mean. The test exploits the property that the above difference is positive for all $x$ for HNBUE alternatives and uses a weighted distance measure. The approach of Klar generalizes an earlier contribution of Jammalamadaka and Lee (1998) who consider a non-weighted distance measure. For a review of earlier contributions, the interested reader is referred to Ascher (1990) and Doksum and Yandell (1984).

\section{The Test Statistics}

To turn to our problem, consider a random sample $X_{1}, \ldots, X_{n}$ from an exponential distribution with density $f(x)=(1 / \theta) \exp \{-x / \theta\}, x>0, \theta>0$ (denote this by $E(\theta)$ ); let $X_{(0)}=0$ and let $X_{(1)}, \ldots X_{(n)}$ denote the order statistics. It is well known that the so-called "normalized spacings"

$$
Y_{i}=(n-i+1)\left(X_{(i)}-X_{(i-1)}\right) \quad i=1, \ldots, n
$$

are again independently and identically distributed (i.i.d.) from $E(\theta)$. Further this property characterizes the exponential distribution, as shown in Seshadri et al (1969). 
There are several inferential procedures based on the normalized spacings. However, our goal in this paper is to use simultaneously the $X$ and the $Y$ variables in order to provide a goodness of fit test for exponentiality.

One of the simplest and natural ways to do this is to compare the empirical distribution function (edf) of the original variables $X$ with that of the transformed ones, $Y$. More precisely, let $F_{n}(t)$ and $G_{n}(t)$ denote the empirical distribution functions of $\left(X_{1}, \ldots, X_{n}\right)$ and $\left(Y_{1}, \ldots, Y_{n}\right)$ respectively. We can construct new tests of exponentiality by measuring the distance between these 2 edf's, using the classical Kolmogorov-Smirnov and Cramer-von Mises type distances. We then obtain the test statistics

$$
T_{1, n}=\sqrt{\frac{n}{2}} \sup _{0 \leq t<\infty}\left|F_{n}(t)-G_{n}(t)\right|
$$

and

$$
T_{2, n}=\frac{n}{2 \bar{X}} \int\left(F_{n}(t)-G_{n}(t)\right)^{2} e^{-t / \bar{X}} d t .
$$

where $\bar{X}$ is the sample mean. Clearly, under the hypothesis of exponentiality, since $\left(X_{1}, \ldots, X_{n}\right)$ and $\left(Y_{1}, \ldots, Y_{n}\right)$ have identical distributions, their empirical distributions $F_{n}(t)$ and $G_{n}(t)$ should be close. Thus one expects $T_{1, n}$ and $T_{2, n}$ to be close to zero under the null hypothesis of exponentiality, while they should be large under any alternative hypothesis.

Although the $X_{1}, \ldots, X_{n}$ and $Y_{1}, \ldots, Y_{n}$ are not independent, our statistics $T_{1, n}$ and $T_{2, n}$ resemble the corresponding two-sample versions and hence the computational formulae for $T_{1, n}$ and $T_{2, n}$ are easily derived. For instance, one computes $T_{1, n}$ just as it is done for the two sample Kolmogorov-Smirnov statistic, using $X_{1}, \ldots, X_{n}$ and $Y_{1}, \ldots, Y_{n}$ as the two "samples." In order to compute $T_{2, n}$, let $Z_{(1)}, \ldots, Z_{(2 n)}$ denote the ordered values obtained by combining $\left\{X_{i}\right\}_{i=1}^{n}$ and $\left\{Y_{i}\right\}_{i=1}^{n}$. Then these $Z_{(i)}$ 's correspond to the jump points of $F_{n}(t)-G_{n}(t)$ and therefore

$$
T_{2, n}=\frac{n}{2} \sum_{i=1}^{2 n-1}\left[F_{n}\left(Z_{(i)}\right)-G_{n}\left(Z_{(i)}\right)\right]^{2}\left[\exp \left\{-Z_{(i)} / \bar{X}\right\}-\exp \left\{-Z_{(i+1)} / \bar{X}\right\}\right]
$$

As shown in the next section, $T_{1, n}$ and $T_{2, n}$ provide "consistent" tests for testing exponentiality, and moreover their null distribution is free of nuisance parameters. These facts, together with their computational simplicity makes them valuable as well as practically useful tests for exponentiality.

\section{$3 \quad$ Asymptotic Properties}

Before we proceed to develop the relevant asymptotic theory for $T_{1, n}$ and $T_{2, n}$ we should note that the two empirical distribution functions are not independent. The 
dependence between the $X$ 's and $Y$ 's can be more easily seen by representing both of them in terms of the spacings $D_{i}=X_{(i)}-X_{(i-1)}$. We can write

$$
F_{n}(t)=\frac{1}{n} \sum_{i=1}^{n} I_{\left\{X_{i} \leq t\right\}}=\frac{1}{n} \sum_{i=1}^{n} I_{\left\{X_{(i)} \leq t\right\}}=\frac{1}{n} \sum_{i=1}^{n} I_{\left\{\sum_{j=1}^{i} D_{j} \leq t\right\}}
$$

and

$$
G_{n}(t)=\frac{1}{n} \sum_{i=1}^{n} I_{\left\{Y_{i} \leq t\right\}}=\frac{1}{n} \sum_{i=1}^{n} I_{\left\{(n-i+1)\left(X_{(i)}-X_{(i-1)}\right) \leq t\right\}}=\frac{1}{n} \sum_{i=1}^{n} I_{\left\{(n-i+1) D_{i} \leq t\right\}}
$$

We first derive some general results on $T_{1, n}$ and $T_{2, n}$.

\subsection{Consistency}

For $0 \leq s \leq 1$, define the two processes

$$
\alpha_{n}(s) \equiv \sqrt{n}\left(F_{n}\left(F_{E}^{-1}(s)\right)-s\right) \quad \text { and } \quad \beta_{n}(s) \equiv \sqrt{n}\left(G_{n}\left(F_{E}^{-1}(s)\right)-s\right),
$$

where $F_{E}$ indicates the distribution function of an $E(\theta)$ r.v.. Then

$$
\sqrt{n}\left(F_{n}(t)-G_{n}(t)\right)=\left[\alpha_{n}\left(F_{E}(t)\right)-\beta_{n}\left(F_{E}(t)\right)\right]
$$

from the continuity of $F_{E}$ it follows that $T_{1, n}$ and $T_{2, n}$ are distribution-free under the null hypothesis of exponentiality. From now on, without loss of generality, we will assume that the random sample $X_{1} \ldots X_{n}$ comes from an $E(1)$ distribution.

Next, we note that by the Glivenko-Cantelli theorem, under the null hypothesis we have that

$$
\sup _{0 \leq t<\infty}\left|F_{n}(t)-F_{E}(t)\right| \stackrel{a . s .}{\rightarrow} 0, \quad \text { and } \sup _{0 \leq t<\infty}\left|G_{n}(t)-F_{E}(t)\right| \stackrel{a . s .}{\rightarrow} 0
$$

from which we easily infer that $T_{1, n} / \sqrt{n} \stackrel{a . s .}{\rightarrow} 0$ and $T_{2, n} / n \stackrel{a . s .}{\rightarrow} 0$ under exponentiality. On the other hand, under the alternative that the observations are distributed according to some continuous d.f. $F_{A}$, following the results in Pyke (1965), we have that

$$
F_{n}(t)-G_{n}(t) \stackrel{a . s .}{\rightarrow} F_{A}(t)-1+\int_{0}^{\infty} f_{A}(y) \exp \left\{-t h_{A}(y)\right\} d y
$$

where $f_{A}(t)$ denotes the density and

$$
h_{A}(t)=\frac{f_{A}(t)}{1-F_{A}(t)}
$$

the hazard rate corresponding to $F_{A}$. It may be recalled that under the null hypothesis of exponentiality this hazard rate, $h_{E}(t)=1$. This indicates that the 2 tests are consistent and their efficiency is tied to the hazard rate of the distribution, a point which will be studied in more detail in the next section. 


\subsection{Asymptotic Null distribution}

Now we consider the problem of determining the asymptotic null distribution of $T_{1, n}$ and $T_{2, n}$. It is well known, see for example Csörgö and Horváth (1993, p.114), that there exist sequences of Brownian Bridges $B_{n}^{1}(t)$ and $B_{n}^{2}(t)$ such that

$$
\sup _{0 \leq t \leq 1}\left|\alpha_{n}(t)-B_{n}^{1}(t)\right| \stackrel{\text { a.s. }}{=} O\left(n^{-1 / 2} \log n\right)
$$

and

$$
\sup _{0 \leq t \leq 1}\left|\beta_{n}(t)-B_{n}^{2}(t)\right| \stackrel{\text { a.s. }}{=} O\left(n^{-1 / 2} \log n\right) .
$$

However, in our case, $\alpha_{n}(s)$ and $\beta_{n}(s)$ are not independent. Therefore, in order to determine the asymptotic behavior of $T_{1, n}$ and $T_{2, n}$, we need to find a joint approximation for the processes $\alpha_{n}(s)$ and $\beta_{n}(s)$. Such joint behavior is discussed in Barbe (1994). It turns out that the two processes are asymptotically independent, which enables us to prove consistency of our test statistics and to study their asymptotic efficiency.

For $0 \leq s, u \leq 1$, let

$$
\begin{gathered}
G_{K}^{\alpha}(s)=\int_{0}^{1}[K(u, s)-s K(u, 1)] d F^{-1}(u) \\
G_{K}^{\beta}(s)=K(s, 1)
\end{gathered}
$$

where $K(.,$.$) denotes a Kiefer process. For details on this process the reader is$ referred to Csörgö and Révész (1981). Note that under exponentiality $F^{-1}(u)=$ $-\log (1-u)$ and $G_{K}^{\alpha}(s)$ and $G_{K}^{\beta}(s)$ have the same distribution as a Brownian Bridge $B(s)$; moreover the joint process $\left(G_{K}^{\alpha}(s), G_{K}^{\beta}(s)\right)$ is Gaussian (Barbe, 1994). Also, since for any choice of $0 \leq s, u, \leq 1$,

$$
\operatorname{Cov}\left(G_{K}^{\alpha}(s), G_{K}^{\beta}(u)\right)=0,
$$

we see that the two processes are independent. We provide the joint approximation in the following

Theorem 1. Under exponentiality one can construct the sequences $\alpha_{n}(s)$ and $\beta_{n}(s)$ and a sequence of Kiefer processes $K_{n}(.,$.$) on the same probability space, such that$

$$
\begin{gathered}
\sup _{0 \leq s \leq 1}\left|\alpha_{n}(s)-G_{K_{n}}^{\alpha}(s)\right|=O_{p}\left(n^{-1 / 2} \phi_{n} \log ^{2} n\right) \\
\sup _{0 \leq s \leq 1}\left|\beta_{n}(s)-G_{K_{n}}^{\beta}(s)\right|=O_{p}\left(n^{-1 / 2} \log ^{2} n\right) .
\end{gathered}
$$


where

$$
\phi_{n}=\left|F^{-1}(1 / n)\right| \vee\left|F^{-1}(1-1 / n)\right|
$$

Proof. The proof is adapted from that of Theorem 2.1 in Barbe (1994) which in turn relies on a result from Komlós et Al. (1975). Consider first the process $\alpha_{n}(s)$, we just need to note that it is equivalent to the process $\alpha_{n}^{U}(s)$ defined in Barbe (1994) and hence we can directly apply the approximation provided therein with the function $\phi_{n}=\log n$ and $F^{-1}(t)=-\log (1-t)$ in the case of exponentiality.

As far as the process $\beta_{n}(s)$ is concerned, we may apply directly the following result of Komlós et Al. (1975) which provides an approximation of the uniform empirical process by a Kiefer process such that

$$
\max _{1 \leq k \leq n} \sup _{0 \leq s \leq 1}\left|k\left(F_{k}(s)-s\right)-K(s, k)\right| \stackrel{\text { a.s. }}{=} O\left(\log ^{2} n\right)
$$

where $F_{k}(s)=k^{-1} \sum_{i=1}^{k} I_{\left\{Y_{i} \leq t\right\}}$. Then, it holds that

$$
\beta_{n}(s)=\frac{K(s, n)}{n^{1 / 2}}+O_{p}\left(n^{-1 / 2} \log ^{2} n\right) .
$$

Reasoning again as in Barbe (1994), we obtain the approximation given in the theorem.

As a consequence of the theorem we see that the processes $\alpha_{n}(s)$, and $\beta_{n}(s)$ are asymptotically independent. From this result consistency and the asymptotic null distribution of $T_{1, n}$ and $T_{2, n}$ follow at once. We state the following

Corollary. Under exponentiality the process $\sqrt{1 / 2}\left[\alpha_{n}(s)-\beta_{n}(s)\right]$ converges weakly to a Brownian Bridge $B(s), 0 \leq s \leq 1$.

Applying Donsker's theorem we obtain the asymptotic distribution of $T_{1, n}$ and $T_{2, n}$.

Theorem 2. Under the null hypothesis of exponentiality,

$$
\begin{gathered}
T_{1, n} \stackrel{\mathcal{D}}{\rightarrow} \sup _{0<s<1}|B(s)|, \\
T_{2, n} \stackrel{\mathcal{D}}{\rightarrow} \int_{0}^{1}|B(s)|^{2} d s,
\end{gathered}
$$

where $B(s), 0 \leq s \leq 1$ denotes a Brownian Bridge. Thus

$$
\lim _{n \rightarrow \infty} P\left(T_{1, n}>t\right)=2 \sum_{k=1}^{\infty}(-1)^{k+1} \exp \left\{-2 k^{2} t^{2}\right\}
$$

and

$$
\lim _{n \rightarrow \infty} P\left(T_{2, n}>t\right)=\frac{1}{\pi} \sum_{k=1}^{\infty}(-1)^{k+1} \int_{(2 k-1)^{2} \pi^{2}}^{(2 k)^{2} \pi^{2}} \frac{1}{y} \sqrt{\frac{-\sqrt{y}}{\sin \sqrt{y}}} \exp \left(-\frac{t y}{2}\right) d y
$$




\section{Approximate Bahadur Efficiency}

Result (1) tells us that the performance of our test statistic is closely connected with the hazard rate of the distribution and this is even more evident if we write the distribution function $F$ in terms of the hazard rate, i.e.

$$
F(t)=1-\exp \left\{-\int_{0}^{t} h(y) d y\right\}
$$

In order to investigate in more depth, we will find the dominant term in (1), for a sequence of alternatives that approach the null hypothesis. To do so, suppose that under the alternative the observations are distributed according to some continuous d.f. $F(t, \theta), \theta \geq 0$ which coincides with the exponential d.f. only for $\theta=0$ and denote, for convenience, the r.h.s. of (1) as $H(t, \theta)$.

We expand formally $H(t, \theta)$ in Taylor series for $\theta$ around $\theta=0$ retaining only the first few terms. To this end, we need some regularity conditions on $F(t, \theta)$ and $f(t, \theta)$ and these are implicit as in e.g. Nikitin (1996). Let the prime denote the derivative with respect to $\theta$, i.e. for any function $g(., \theta)$

$$
g^{\prime}(., \theta)=\frac{\partial g(., \theta)}{\partial \theta}
$$

If we compute the derivative of the function $H(t, \theta)$ wrt $\theta$ we obtain

$H^{\prime}(t, \theta)=F^{\prime}(t, \theta)+\int_{0}^{\infty} f^{\prime}(y, \theta) \exp \{-t h(y, \theta)\} d y-t \int_{0}^{\infty} f(y, \theta) h^{\prime}(y ; \theta) \exp \{-t h(y, \theta)\} d y$.

To evaluate $H^{\prime}(t, 0)$ we recall that $h(t, 0)=h_{E}(t)=1$ and note that, by definition and regularity conditions

$$
h^{\prime}(t, 0)=e^{t}\left[f^{\prime}(t, 0)+F^{\prime}(t, 0)\right], \quad \int_{0}^{\infty} f^{\prime}(t, 0) d t=0 .
$$

After some simplifications we obtain

$$
H^{\prime}(t, 0)=F^{\prime}(t, 0)-t e^{-t} \int_{0}^{\infty} F^{\prime}(y, 0) d y .
$$

Together with the fact that $H(t, 0)=0$ we finally have that

$$
H(t, \theta)=\left[F^{\prime}(t, 0)-t e^{-t} \int_{0}^{\infty} F^{\prime}(y, 0) d y\right] \theta+O\left(\theta^{2}\right)
$$

This result may be used to compute the approximate local Bahadur slope in order to make some comparisons with other tests statistics efficiencies. Recall that if a 
sequence $\left\{T_{n}\right\}$ satisfies $T_{n} / \sqrt{n} \stackrel{P_{\theta}}{\rightarrow} b(\theta), \quad \theta>0$ and, under the null hypothesis, the limiting distribution $F(t)=\lim _{n \rightarrow \infty} P\left(T_{n} \leq t\right)$ satisfies $\log [1-F(t)]=-\frac{a t^{2}}{2}(1+o(1))$, as $t \rightarrow \infty$, then the approximate Bahadur slope of the standard sequence $T_{n}$ is defined as $c_{T}(\theta)=a[b(\theta)]^{2}$.

Given the asymptotic independence of the processes based on the observations and the normalized spacings and result (1) it is possible to compute the approximate Bahadur slope for the two statistics $T_{1, n}$ and $T_{2, n}$ (we take the root of $T_{2, n}$ in order to have a normalized sequence). The value of the constant $a$ may be determined from the corresponding results for two samples Kolmogorov Smirnov and Cramer-von Mises statistics, they can be found, for example in Nikitin (1995). Let $c_{T_{1, n}}(\theta)$ and $c_{T_{2, n}}(\theta)$ denote the approximate Bahadur slopes of $T_{1, n}$ and $T_{2, n}$ respectively. Then

$$
c_{T_{1, n}}(\theta)=\left[\sup _{0 \leq t \leq \infty}|H(t, \theta)|\right]^{2}
$$

and

$$
c_{T_{2, n}}(\theta)=\frac{\pi^{2}}{4}\left[\int_{0}^{\infty} H(t, \theta)^{2} e^{-t} d t\right]
$$

Next, we can use result (2) to compute the approximate slope as $\theta \rightarrow 0$. We now compare these approximate Bahadur slopes of the statistics $T_{1, n}$ and $T_{2, n}$ with those of Kolmogorov-Smirnov and Cramer-von Mises one sample statistics for the simple hypothesis of exponentiality where the mean is given. It should be remarked that these are purely for the sake of comparison since there is no hope that our tests for the composite hypothesis of exponentiality, will beat the latter tests. We utilize the Linear Failure Rate, Makeham, and Weibull alternatives, which are often considered in the evaluation of the performance of tests for exponentiality (Doksum and Yandell (1984), Nikitin (1996)).

Example 1. Consider a distribution with density $f(t, \theta)=(1+\theta t) \exp \left\{-t-(1 / 2) \theta t^{2}\right.$ (linear failure rate). In this case we have

$$
F^{\prime}(t, 0)-t e^{-t} \int_{0}^{\infty} F^{\prime}(y, 0) d y=\frac{1}{2} t e^{-t}(t-2) .
$$

From this we obtain that $c_{T_{1, n}}(\theta)=0.0532 \theta^{2}$ and $c_{T_{2, n}}(\theta)=0.061 \theta^{2}$ as $\theta \rightarrow 0$. To obtain the corresponding results for one sample $\mathrm{KS}$ and $\omega^{2}$ statistics we expand $F(t, \theta)$ around $\theta=0$ to obtain

$$
F(t, \theta)-F(t, 0)=F^{\prime}(t, 0) \theta+O\left(\theta^{2}\right)
$$

Applying standard techniques for computing the approximate slope of these statistics (see, e.g. Nikitin 1996) we obtain $c_{K S}(\theta)=0.2930 \theta^{2}$ and $c_{\omega^{2}}(\theta)=0.2437 \theta^{2}$ as $\theta \rightarrow 0$. 
From this we see that the relative efficiency are $e_{T_{1, n}, K S}^{B}=c_{T_{1, n}}(\theta) / c_{K S}(\theta) \simeq 0.18$ and $e_{T_{2, n}, \omega^{2}}^{B}=c_{T_{2, n}}(\theta) / c_{\omega^{2}}(\theta) \simeq 0.21$

Example 2. Take a Makeham density $f(t, \theta)=\left[1+\theta\left(1-e^{-t}\right)\right] \exp \{-[t+\theta(t+$ $\left.\left.\left.e^{-t}-1\right)\right]\right\}$, from

$$
F^{\prime}(t, 0)-t e^{-t} \int_{0}^{\infty} F^{\prime}(y, 0) d y=e^{-t}\left[e^{-t}+t / 2-1\right]
$$

we have $c_{T_{1, n}}(\theta)=0.0077 \theta^{2}$ and $c_{T_{2, n}}(\theta)=0.008 \theta^{2}$ as $\theta \rightarrow 0$. Also, $c_{K S}(\theta)=0.1048 \theta^{2}$ and $c_{\omega^{2}}(\theta)=0.1 \theta^{2}$ as $\theta \rightarrow 0$, and hence $e_{T_{1, n}, K S}^{B}=\simeq 0.07$ and $e_{T_{2, n}, \omega^{2}}^{B} \simeq 0.08$.

Example 3. Consider a Weibull density of the form $f(t, \theta)=(\theta+1) t^{\theta} \exp \left\{-t^{1+\theta}\right\}$.

$$
F^{\prime}(t, 0)-t e^{-t} \int_{0}^{\infty} F^{\prime}(y, 0) d y=t e^{-t}[\log t+\gamma-1]
$$

where $\gamma$ is Euler's constant. It turns out that $c_{T_{1, n}}(\theta)=0.1321 \theta^{2}$ and $c_{T_{2, n}}(\theta)=$ $0.1377 \theta^{2}$ as $\theta \rightarrow 0$. Also, $c_{K S}(\theta)=0.2916 \theta^{2}$ and $c_{\omega^{2}}(\theta)=0.3113 \theta^{2}$ as $\theta \rightarrow 0$, and hence $e_{T_{1, n}, K S}^{B}=\simeq 0.45$ and $e_{T_{2, n}, \omega^{2}}^{B} \simeq 0.44$.

We note that the approximate slopes of $T_{1, n}$ and $T_{2, n}$ are always close to each other, but fall short of the classical Kolmogorov-Smirnov and $\omega^{2}$ one sample statistics with known mean. This is to be expected in view of the fact that the slopes for classical Kolmogorov-Smirnov and $\omega^{2}$ one sample statistics are computed under a simple hypothesis of exponential with given mean, whereas for our statistics the slope has been calculated under the composite hypothesis where the mean is not specified. Note, in fact, that there is no need to estimate the mean in computing $T_{1, n}$; a version of the quadratic statistics $T_{2, n}$ with analogue characteristics could be developed also. Further, approximate Bahadur slopes are only a rough measure of the asymptotic efficiency. In the next section we examine some simulated power values of our statistics.

\section{Monte Carlo Power Comparisons}

In this section we are going to compare the power performance of $T_{1, n}$ and $T_{2, n}$ with those of other tests statistics that have appeared in the literature. In particular, we consider traditional one sample Kolmogorov Smirnov and $\omega^{2}$ statistics with estimated parameters and other tests based on a characterization of the exponential distribution that have been proposed in the literature such as those proposed by Angus (1982) and based on a loss of memory type functional equation (actually on an order statistics characterization) but also, on this subject one can consult Ahmad and Alwasel (1999) which propose a different test statistics based on the same functional equation. Next, we consider the proposal of Baringhaus and Henze (2000) which define Kolmogorov 
Smirnov and Cramer von Mises type statistics relying on a characterization via mean residual life. The last two alternative statistics considered are those of Ebrahimi et Al. (1992) (but see also Grzegorzewski and Wieczorkowski, (1999)) whose test is based on entropy and Gail and Gastwirth (1978) which propose a test for exponentiality based on the Gini's index which is constructed from the area under the Lorenz curve; this test, also in the light or the recent results of Nikitin and Tchirina (1996) is a test which performs well in a variety of situations. Table I summarizes the relevant information about the alternative test statistics used.

Table I. Goodness of fit tests studied.

\begin{tabular}{l|l|l} 
Symbol & Definition / Characterization & Reference \\
\hline KS & Two sided Kolmogorov Smirnov test with estimated mean & Durbin (1975) \\
$\omega^{2}$ & Cramer von Mises statistic with estimated mean & Darling (1957) \\
$A_{1, n}$ & KS type based on Loss of memory functional equation & Angus (1982) \\
$A_{2, n}$ & CVM type based on Loss of memory functional equation & Angus (1982) \\
$H_{1, n}$ & KS type based on Mean Residual life function & Baringhaus \& Henze (2000) \\
$H_{2, n}$ & Cramer Von Mises type based on Mean Residual life & Barighaus \& Henze (2000) \\
$V_{m, n}$ & Based on Vasicek's (1976) entropy estimator & Ebrahimi et Al. (1992) \\
Gini & Based on Gini's index & Gail \& Gastwirth (1978) \\
\hline
\end{tabular}

As far as the alternative distributions considered in the Monte Carlo experiment, they are reported in Table II. We have tried to encompass some common distributions frequently considered in other power studies as well as to have a certain variety of life distributions differing from the point of view of the hazard rate. The choice of the parameters has been made in such a way that the resulting density is not too far away in shape from the exponential one.

Table II. Alternative distributions considered.

\begin{tabular}{l|l|l|l} 
Symbol & $F(t)[f(t)$ when indicated $]$ & Support & Failure Rate \\
\hline Pareto & $1-[(\theta-2) /(\theta-1)]^{\theta-1} t^{1-\theta}$ & $t \geq \theta-2) /(\theta-1)$ & Decreasing \\
Weibull & $1-\exp \left\{-t^{\theta}\right\}$ & $t \geq 0$ & Increasing $\theta>1$, \\
& & & Decreasing $\theta<1$ \\
Log Normal & $f(t)=\frac{1}{\sqrt{2 \pi} t \theta} \exp \left\{\log ^{2}(t) /\left(2 \theta^{2}\right)\right\}$ & $t \geq 0$ & Hump Shaped \\
Shifted Exponential & $1-\exp \{-(t-\theta)\}$ & $t \geq \theta$ & Constant \\
Linear Failure Rate & $1-\exp \left\{-t-\theta t^{2} / 2\right\}$ & $t \geq 0$ & Increasing \\
Dhillon & $1-\exp \left\{1-e^{t^{\theta}}\right\}$ & $t \geq 0$ & Bathtub Shaped, $\theta<1$ \\
\hline
\end{tabular}

In the next table we find the power estimates for sample of moderate size $(n=20)$ for the goodness fit tests considered in Table I and the alternative distributions of Table II. The estimates have been obtained by rounding to the nearest integer the percentage of statistics declared significant out of 10,000 samples of size 20. In order to check our routines, we have reproduced the power studies contained in the original 
papers of Table I, obtaining substantially equal results. The test statistics of Ebrahimi et Al. (1992) required the choice of a window parameter $m$. Also in the light of Taufer (2002) we chose a value $m=4$ for all situations.

Table III. Monte Carlo Power estimates based on 10,000 samples of size 20. Significance level $\alpha=0.05$.

\begin{tabular}{l|l|l|l|l|l|l|l|l|l|l} 
Distribution & $T_{1, n}$ & $\mathrm{KS}$ & $A_{1, n}$ & $H_{1, n}$ & $V_{4, n}$ & Gini & $T_{2, n}$ & $\omega^{2}$ & $A_{2, n}$ & $H_{2, n}$ \\
\hline Pareto(2.2) & 87 & 93 & 99 & 83 & 99 & 44 & 67 & 91 & 97 & 74 \\
Pareto(2.5) & 97 & 98 & 99 & 95 & 99 & 53 & 91 & 98 & 99 & 86 \\
Weibull(0.8) & 04 & 17 & 02 & 13 & 04 & 24 & 04 & 20 & 04 & 21 \\
Weibull(1.4) & 27 & 29 & 25 & 36 & 37 & 36 & 26 & 35 & 29 & 37 \\
LogNormal(0.6) & 73 & 84 & 81 & 84 & 90 & 80 & 74 & 89 & 84 & 84 \\
LogNormal(0.8) & 28 & 30 & 42 & 29 & 42 & 24 & 26 & 34 & 38 & 27 \\
Shifted Exp(0.2) & 28 & 25 & 42 & 23 & 59 & 22 & 25 & 28 & 34 & 23 \\
LFR(0.5) & 12 & 10 & 09 & 14 & 13 & 10 & 12 & 11 & 11 & 11 \\
LFR(3.0) & 30 & 30 & 23 & 39 & 36 & 38 & 30 & 37 & 29 & 38 \\
Dhillon(0.7) & 06 & 06 & 03 & 06 & 05 & 05 & 06 & 06 & 05 & 05 \\
Dhillon(0.9) & 17 & 15 & 10 & 21 & 20 & 17 & 17 & 17 & 14 & 18 \\
\hline
\end{tabular}

This power study provides some elements to make an overall comparison among tests for exponentiality based on characterizations. First of all, we note that there is little or no difference between the powers of the tests $T_{1, n}$ and $T_{2, n}$, so we may mention $T_{1, n}$ only in our reasoning. The test does not perform as well as most of its competitors for Weibull alternatives with $\theta<1$ (and also Gamma). Actually, the same happens to the test statistics $A_{1, n}$ and $A_{2, n}$ based on the loss of memory characterization. Apart from this case, the test statistics $T_{1, n}$ and $T_{2, n}$ compare well with most of the other tests although they do not uniformly outperform any of the other tests for the cases considered. Although there is clearly not a best test from the point of view of power for all situations, we see that the statistics based on mean residual life and entropy show good power for most alternatives.

\section{$6 \quad$ Examples}

As examples of application we use the data set given in Grubbs (1971) and Wadsworth (1990, p.611), these data sets have been used in Ebrahimi et Al. (1992), Ahmad and Alwasel (1999) and Shapiro (1995) to show the application of various tests for exponentiality. The first data set (Table IV) are the times between arrivals of 25 customers at a facility, their quantile plot show a clear departure from the exponentiality hypothesis, the hypothesis of exponentiality is rejected with $p$-value smaller that 0.01 by all the tests considered in Shapiro (1995). The second data set (Table V) represents mileages for 19 military personal carriers that failed in service, these data have been used by Ebrahimi et Al. (1992) and Ahmad and Alwasel (1999), they come to the conclusion that the hypothesis of exponentiality is tenable in this case. 
Let us begin with the data set of Wadsworth (1990), the inter arrival times have been ordered in Table IV.

Table IV: Inter arrival times

\begin{tabular}{lllllllllllll}
\hline 1.80 & 2.89 & 2.93 & 3.03 & 3.15 & 3.43 & 3.48 & 3.57 & 3.85 & 3.92 & 3.98 & 4.06 & 4.11 \\
4.13 & 4.16 & 4.23 & 4.34 & 4.37 & 4.53 & 4.62 & 4.65 & 4.84 & 4.91 & 4.99 & 5.17 & \\
\hline
\end{tabular}

From the data we obtain $T_{1, n}=2.83$ and $T_{2, n}=2.87$. Both statistics reject the hypothesis of exponentiality with an estimated $p$-value well beyond the 0.001 level.

As far as the second data set is concerned, the mileages have been ordered in Table V.

Table V: Mileages.

\begin{tabular}{rrrrrrrrrr}
\hline 162 & 200 & 271 & 320 & 393 & 508 & 539 & 629 & 706 & 778 \\
884 & 1003 & 1101 & 1182 & 1463 & 1603 & 1984 & 2355 & 2880 & \\
\hline
\end{tabular}

Performing the computations we have the values $T_{1, n}=0.65$ with an estimated $p$-value of $0.44 T_{2, n}=0.1283$ with an estimated $p$-value of 0.18 .

Acknowledgements. The authors would like to thank a referee who pointed out a critical error in our earlier draft and helped improve the paper's readability.

\section{REFERENCES}

Alwasel,I. (2001). On goodness of fit testing for exponentiality using the memoryless property. J. of Nonparametric Statistics 13, 569-581.

Angus, J.E. (1982). Goodness of fit tests for exponentiality based on a loss of memory type functional equation. J.Stat. Planning and Inference 6, 241-251.

Barbe, P. (1994). Joint approximation of processes based on spacings and order statistics. Stochastic processes and their applications 53, 339-349.

Baringhaus, L. and Henze, N. (1991). "A class of consistent tests for exponentiality based on the empirical Laplace transform," Ann. Inst. Statist. Math. 43, 551-564.

Baringhaus,L. and Henze,N. (1992). "An Adaptive Omnibus Test for Exponentiality," Communications in Statistics - Theory and Methods 21, 969-978.

Baringhaus, L. and Henze, N. (2000). "Tests of fit for exponentiality based on a characterization via the mean residual life function," Statistical Papers 41, 225-236.

Csörgö,M. and Horváth, L. (1993). Weighted approximations in probability and statistics. Wiley, New York.

Csörgö,M. and Révész, P. (1981). Strong approximations in probability and statistics. Academic Press, Budapest. 
Darling, D. (1957). The Kolmogorov Smirnov Cramer von Mises tests. Ann. Math. Stat. 28, 823-838.

Doksum, K.A. e Yandell, B.S. (1984). "Tests for exponentiality," Handbook of statistics, Vol. 4, North Holland, 579-612.

Durbin, J. (1975). Kolmogorov Smirnov tests when parameters are estimated with applications to tests to exponentiality and tests on spacings. Biometrika, 62, 5-22.

Ebrahimi, N., Soofi, E.S. and Habibullah, M. (1992). "Testing Exponentiality Based on Kullback-Leibler Information," Journal of the Royal Statistical Society B 54, 739-748.

Gail, M.H. and Gastwirth, J.L. (1978). "A Scale Free Goodness of Fit Test for the Exponential distribution based on the Gini statistic" Journal of the Royal Stat. Soc. 40, 350357.

Grzegorzewski, P. and Wieczorkowski, R. (1999). "Entropy-Based Goodness of Fit Test for Exponentiality," Communications in Statistics - Theory and Methods 28, 1183-1202.

Grubbs, F.E. (1971) Fiducial bounds on reliability for the two parameter negative exponential distribution. Technometrics 13, 873-876.

Henze, N. (1993). "A New Flexible Class of Omnibus Tests for Exponentiality," Communications in Statistics - Theory and Methods 22, 115-133.

Jammalamadaka,S.R. and Lee,E.S. (1998). "Testing for Harmonic New Better than Used in Expectation", Probab. Eng. Inform. Sci. 12, 409-416.

Klar, B. (2000). "A class of tests for exponentiality against HNBUE alternatives," Statistics and Prob. Letters 47, 199-207.

Komlós, J., Major, P. and Tusnády (1975). An approximation of partial sums of independent R.V.'s and the sample D.F. Zeit. Wahrsch. Verv. Gebiete 32, 111-131. Nikitin, Y.Y. (1995). Asymptotic efficiency of nonparametric tests. Cambridge University Press, New York.

Nikitin, Y.Y. (1996). Bahadur efficiency of a test of exponentiality based on a loss of memory type functional equation. J. of Nonparametric Statistics 6, 13-26.

Nikitin, Y.Y. and Tchirina, A.V. (1996). Bahadur efficiency and local optimality of a test for the exponential distribution based on the Gini statistic. J. Ital. Stat. Soc. 1, 163-175.

Pyke, R. (1965). Spacings. J. Royal Stat. Soc. 3, 395-436.

Seshadri, V., Csörgő, M. and Stephens, M.A. (1969). "Tests for the Exponential Distribution using Kolmogorov-type Statistics," Journal of the Royal Statistical Society B 31, 499-509. 
Shapiro, S.S. (1995). Goodness of Fit tests. In The Exponential Distribution: Theory Methods and Applications. Balakrishnan, N. and Basu, A.P. Editors, Gordon and Breach, Amsterdam.

Taufer, E. (2000). A new test for exponentiality against omnibus alternatives. Stochastic Modeling and Applications 3, 23-36.

Taufer, E. (2002). On entropy based tests for exponentiality. Communications in Statistics - Simulation and Computation, 31, 189-200. 